\title{
QUE PEUVENT FAIRE LES VILLES POUR FAVORISER LA CONCILIATION TRAVAIL-FAMILLE ET LE BIEN-ÊTRE DES CITOYENS ?
}

\author{
Juliette Rochman et Diane-Gabrielle Tremblay \\ HEC Montréal | Gestion
}

2012/4 - Vol. 37

pages 34 à 44

ISSN 0701-0028

Article disponible en ligne à l'adresse:

http://www.cairn.info/revue-gestion-2012-4-page-34.htm

Pour citer cet article :

Rochman Juliette et Tremblay Diane-Gabrielle, « Que peuvent faire les villes pour favoriser la conciliation travail-famille et le bien-être des citoyens ? »,

Gestion, 2012/4 Vol. 37, p. 34-44. DOI : 10.3917/riges.374.0034

Distribution électronique Cairn.info pour HEC Montréal.

(c) HEC Montréal. Tous droits réservés pour tous pays.

La reproduction ou représentation de cet article, notamment par photocopie, n'est autorisée que dans les limites des conditions générales d'utilisation du site ou, le cas échéant, des conditions générales de la licence souscrite par votre établissement. Toute autre reproduction ou représentation, en tout ou partie, sous quelque forme et de quelque manière que ce soit, est interdite sauf accord préalable et écrit de l'éditeur, en dehors des cas prévus par la législation en vigueur en France. II est précisé que son stockage dans une base de données est également interdit. 


\section{Dossier : Conciliation travail-famille}

\section{QUE PEUVENT FAIRE LES VILLES POUR FAVORISER LA CONCILIATION travail-famille et le bien-être des citoyens?}

Par Juliette Rochman et Diane-Gabrielle Tremblay

\section{RÉSUMÉ}

De nombreux changements environnementaux, comme les pressions démographiques et concurrentielles jumelées avec l'étalement urbain, exercent une influence sur la conciliation travail-famille et sur le bien-être des personnes, en particulier dans les grandes villes des pays industrialisés. Au cours des dernières années, certaines municipalités et collectivités à travers le monde ont lancé des initiatives visant à adopter des pratiques facilitant l'articulation entre vie professionnelle et vie personnelle. Se basant sur des entrevues menées auprès de dirigeants de collectivités françaises et sur une synthèse des écrits sur le sujet, cet article présente une typologie des pratiques que les collectivités peuvent mettre en œuvre et les conditions à respecter pour améliorer leurs retombées. 


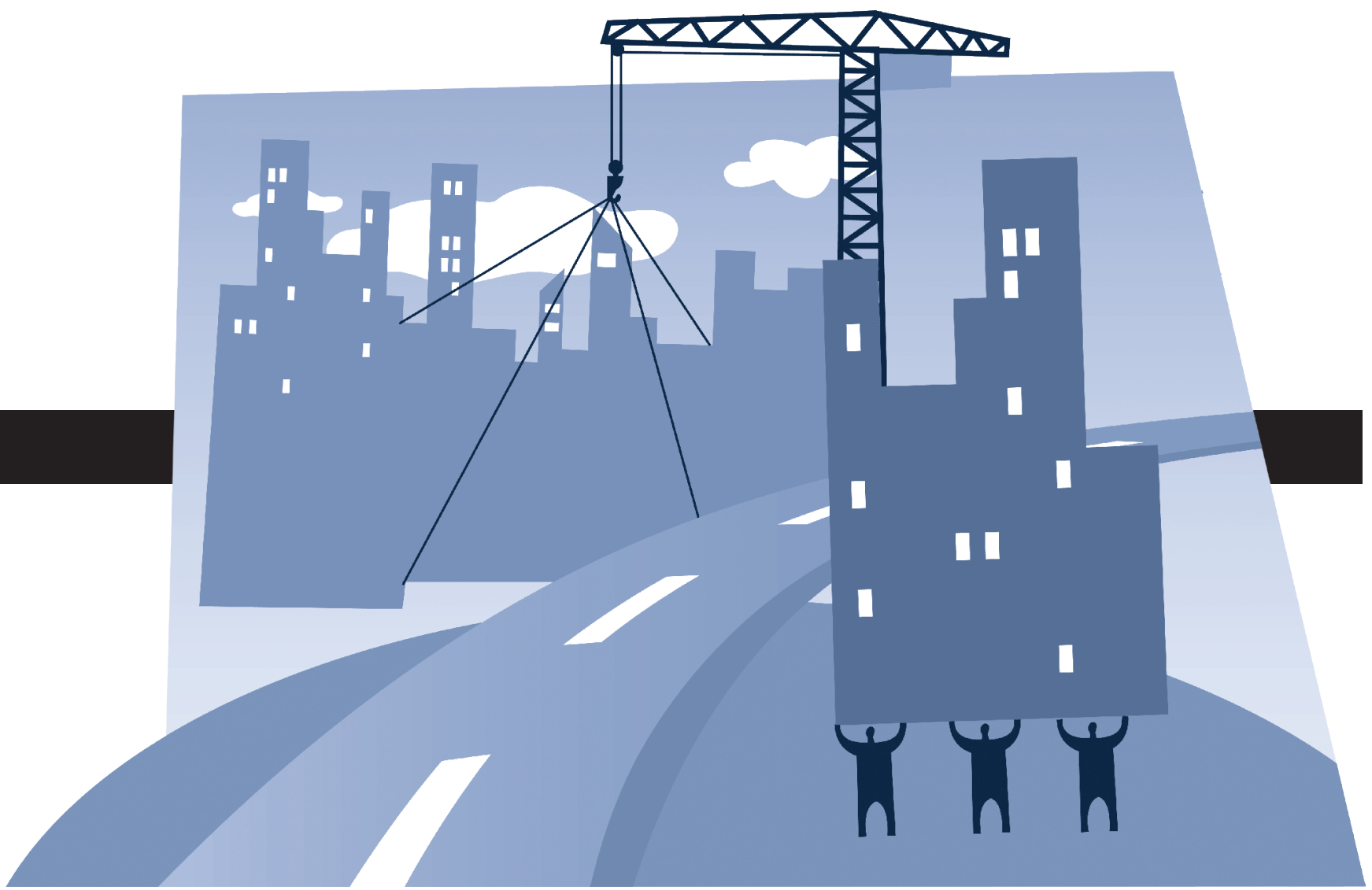

La conciliation travail-famille fait l'objet de préoccupations dans de nombreux pays. Les pays européens, notamment, connaissent sensiblement les mêmes changements socioéconomiques et démographiques que le Québec ${ }^{1}$. En effet, dans le contexte économique et social actuel, la majorité des familles ont besoin de deux revenus pour faire face aux impératifs financiers de la vie. De même, le rythme accéléré auquel les femmes se sont intégrées au marché du travail, l'augmentation de la proportion de personnes âgées, la lenteur avec laquelle les organisations sociales et économiques s'adaptent aux nouvelles réalités des familles ainsi que les exigences du marché du travail sont autant de facteurs qui exacerbent les difficultés de conciliation entre les activités et les obligations liées aux sphères professionnelle et personnelle.

La conciliation travail-famille, sur laquelle on se penche en Europe comme au Québec ${ }^{2}$, est traitée dans le cadre de nombreuses politiques et initiatives nationales de plus en plus souvent appliquées à l'échelon local. Au Québec, les politiques familiales municipales ont apporté des pistes de solution en partant d'une approche centrée sur la famille, son cadre de vie et l'épanouissement de chacun de ses membres. Bien qu’originale, cette approche ne se démarque pas encore d'une logique de planification des villes et des services basée sur l'espace, alors que s'impose de plus en plus une planification temporelle des activités et des services.

C'est en cela que réside l'originalité de l'expérience des «bureaux des temps» que nous décrivons dans cet article. Nous nous sommes plus spécifiquement intéressées au cas de la France, dans la mesure où, même si les bureaux des temps ne disposent plus d'un soutien de la part de l'État (depuis 2001) et ne bénéficiant pas d'un cadre législatif (comme en Italie), ils se sont multipliés et ont connu beaucoup de succès.

À travers l'analyse de l'évolution récente (2007-2010) des politiques temporelles en France (et dans une moindre mesure ailleurs en Europe) et de leurs conditions de succès, nous fournissons des pistes de réflexion sur le potentiel et la portée actuelle de ces démarches. Cela est de nature à intéresser diverses collectivités québécoises (les municipalités, les Conférences régionales des élus, les municipalités régionales de comté), car nombre d'entre elles commencent à intervenir dans ce domaine et cherchent à agir sur leur territoire en matière de conciliation travail-famille, éventuellement pour implanter des bureaux des temps ou des initiatives apparentées. 


\section{LES POLITIOUES TEMPORELLES}

\section{en Europe}

C’est en Italie, à la fin des années 1980, qu’ont été élaborées les premières politiques temporelles en Europe. Elles ont été appuyées par des mouvements féministes (qui revendiquaient le droit de concilier les divers temps de leur vie, ou temps sociaux) et par des syndicats. Elles ont été consacrées par deux lois : la loi 142, en 1990, qui donne aux maires italiens la responsabilité de la gestion des horaires des services publics sur leur territoire, et la loi TURCO, en 2000, qui "promeut un équilibre entre les temps de travail, de loisirs, de formation et de sociabilité à travers l'institution de congés parentaux et de congés pour formation continue, de coordination des temps de fonctionnement de la ville et de promotion des usages du temps à des fins de solidarité sociale».

Très rapidement, la problématique, d'abord centrée en Italie sur les temps de la personne, sélargit à l'espace public. S'inscrivant dans une optique de revitalisation urbaine, son objectif est notamment de déterminer comment il est possible de créer, avec les habitants d'un quartier, un «espacetemps» convivial en réhabilitant une place publique. Il s'agit également d'aborder le problème de la mobilité en tentant, par exemple, d'améliorer la circulation de la ville de Crémone à travers un "plan de mobilité » en collaboration avec les entreprises, la municipalité et les citoyens.

Les politiques temporelles font leur apparition à peu près simultanément dans plusieurs pays en partie sous l'impulsion d'un programme européen sur l'excellence territoriale en Europe (EUREXCTER).

Au Québec, il y a eu des discussions sur l'établissement d'un bureau des temps dans la ville de Québec, mais celui-ci n'a pas vu le jour. Par contre, dans le cadre des politiques familiales municipales ${ }^{3}$, plusieurs villes s'intéressent à des sujets connexes, sans toutefois avoir une politique bien arrêtée en la matière ${ }^{4}$. Enfin, plus récemment, la municipalité régionale de comté (MRC) de Charlevoix-Est a mis sur pied une agence des temps, quelle présente comme «un bureau des temps typiquement québécois ${ }^{5} »$. La thématique suscite de plus en plus d'intérêt, d'où la pertinence de notre recherche sur ses origines et son développement.

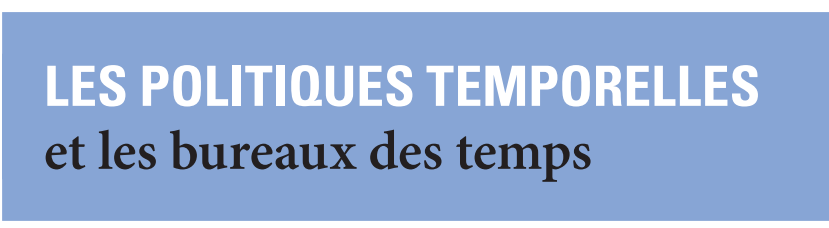

Les politiques temporelles de la ville ont pour objectifs tant l'amélioration de la qualité de la vie des citoyens que l'égalité entre les sexes, les âges et les catégories sociales, la reconstitution du lien social et la revitalisation urbaine ${ }^{6}$. Un des enjeux consiste à concilier les activités quotidiennes dans différentes sphères - le travail, la famille, les activités sociales et les loisirs. Les politiques temporelles résultent également de la multiplication et de la diversification des usages du temps, ainsi que de la sensibilité croissante des populations à l'égard des questions relatives au temps.

Un nouveau type de structure accompagne la mise en œuvre des politiques temporelles : le bureau des temps, parfois appelé «mission des temps» ou «agence des temps». «Il s'agit d'une structure, municipale ou fortement articulée à laction de la collectivité territoriale, qui assure cette transversalité tout en étant à l'écoute des habitants, des utilisateurs du territoire, en même temps qu'elle les associe à la formulation des problèmes et des solutions ${ }^{7}$.» Dans ce dialogue sociétal et avec l'aide des bureaux des temps doivent sétablir les compromis destinés à résoudre les conflits temporels inhérents aux sociétés individualisées et diversifiées, entre autres en matière de conciliation travail-famille ${ }^{8}$.

Au-delà des particularités et des priorités nationales et locales, un consensus s'est progressivement créé entre les acteurs des politiques temporelles, à léchelle de l'Europe, autour de quelques thématiques et champs d'intervention. Il ressort différents enjeux abordés à la fois de façon systématique et différenciée par les politiques temporelles et comportant des dimensions individuelle et collective.

Létablissement d'un relatif consensus autour de ces enjeux, leur intégration dans des politiques temporelles et la mise en œuvre des expériences locales se sont traduits par la constitution d'un «corpus assez homogène de méthodes,

Les politiques temporelles de la ville ont pour objectifs tant l'amélioration de la qualité de la vie des citoyens que l'égalité entre les sexes, les âges et les catégories sociales, la reconstitution du lien social et la revitalisation urbaine. Un des enjeux consiste à concilier les activités quotidiennes dans différentes sphères - le travail, la famille, les activités sociales et les loisirs. 


\section{Les Bureaux des temps en pratique}

\section{Bureau - mission -ou agence des temps?}

"Un bureau c'est quelque chose qui est visible du point de vue administratif. Un bureau en administration centrale signifie qu'il y a une mission avec des gens derrière et dans une collectivité territoriale (bureau du droit des sols) cela correspond à des missions précises, publiques, claires reconnues. De ce point de vue, Paris a un bureau des temps, inscrit au sein de la direction des services publics et de la communication. " P. V (St Denis entrevue 2010)

Mais certaines collectivités ont délaissé le terme de bureau des temps pour d'autres termes. L'objectif était de montrer que le bureau des temps dépassait le cadre strictement administratif, que leur centre de préoccupation ne se limitait pas à des questions d'horaires mais comprenait également une dimension géographique ou territoriale et accordait une place prépondérante au dialogue social et sociétal. Ces collectivités ont donc retenu l'appellation d'espace des temps, comme à Lyon ou à St Denis.

\section{À quelle échelle penser un bureau des temps?}

II n'existe pas de règle définissant l'échelle la plus appropriée pour l'implantation d'un bureau des temps (ville, agglomération, département). Il est toutefois impossible de faire de la politique temporelle tout seul dans son coin. Il faut au moins avoir un minimum de champ d'action. Le grand Lyon c'est cohérent. Paris, c'est Paris. " (J-Y Boulin entrevue 2010)

Au Québec, on peut imaginer que les plus grandes villes, comme Montréal, Québec et Gatineau, pourraient développer leur propre bureau des temps, ou agence semblable, mais auraient sans doute intérêt aussi à se concerter à l'échelle de la communauté métropolitaine. Dans d'autres territoires, ce serait la MRC (Municipalité régionale de comté) qui serait sans doute la plus apte à intervenir en la matière. De fait, la MRC de Charlevoix-est a créer une Agence des temps.

\section{S'appuyer sur la gouvernance locale : une clé du succès}

La construction de formes de gouvernance incluant à la fois les acteurs des secteurs publics (et les employés de ce secteur), privé, et civil est fondamentale à plusieurs titres. Elle constitue l'essence même de la logique temporelle qui sous-tend les bureaux des temps. Ceux-ci sont en effet conçus comme des relais permettant la diffusion d'une politique et de démarches ascendantes favorisant la prise en compte et l'intégration des temporalités et des rythmes individuels et collectifs dans l'aménagement du territoire. Les tables de concertation "quadrangulaires" du modèle italien relèvent de cette logique et l'on retrouve au sein des focus groupes et des comités de pilotage des bureaux des temps français cette composition.

Cette construction est également déterminante d'un point de vue opérationnel car plupart du temps, le bureau des temps ne peut porter, et encore moins financer, seul le projet (et il n'a d'ailleurs pas cette vocation). La mission consiste à favoriser la concertation et à impliquer les acteurs les plus directement concernés afin qu'ils soient en mesure de reprendre en charge la responsabilité du dossier. Dans la plupart des cas observés, un projet émerge à l'initiative du bureau des temps et/ou des acteurs locaux, une concertation a lieu avec les différents groupes d'acteurs privés et institutionnels (tels I'ADEME, la CAF, la SNCF ou d'autres sociétés de transport). A partir de là, le bureau des temps réalise un accompagnement du projet jusqu'à ce que celui-ci soit suffisamment développé pour être repris en charge par le principal service concerné.

d'outils et de processus ${ }^{9} »$. Ces méthodes comportent une base commune articulant quatre séries d'actions.

Premièrement, la sensibilisation constitue une des clés de la faisabilité des politiques temporelles. Il faut sensibiliser les élus, les acteurs territoriaux amenés à appliquer les politiques temporelles ainsi que les personnes et les groupes cibles de ces politiques (dans la mesure où l'on connaît ceuxci). Le but de la sensibilisation est de montrer l'importance des problématiques abordées par les politiques temporelles et les bureaux des temps et l'intérêt des actions menées ailleurs et à mener localement. Il est nécessaire de mieux expliquer ce qu'est un bureau des temps, autant dans la collectivité qu'aux différents partenaires. On peut penser qu'un bureau des temps consiste uniquement à augmenter l'ouverture des équipements, alimentant le cycle suivant : bureau des temps - horaires - amplitude horaire - détérioration des conditions de travail. Il faut dépasser ces préjugés car le but de la démarche est précisément l'inverse, soit améliorer les conditions de vie et de travail.

Il importe donc de s'appuyer sur une réalité, notamment sur les réalisations d'autres bureaux des temps. Il faut ainsi faire des rappels réguliers (des réunions avec les services de concertation, par exemple) et travailler auprès de l'interne et de l'externe. La diffusion des expériences locales peut être favorisée, au-delà du travail individuel des élus et des chargés de mission, par le travail de veille effectué par le réseau Tempo territorial ${ }^{10}$, une association regroupant la plupart des collectivités s'étant dotées d'une politique temporelle. Créée en 2004 pour pallier le manque de soutien de l'État et la forte probabilité d'un nouveau tarissement du 
financement européen, l'association a produit nombre de documents de vulgarisation sur les pratiques temporelles et réalisé des débats thématiques bimensuels, «les mardis de Tempo », sur des questions de société liées au temps, telles que la nuit, les dimanches et l'urbanisme.

Deuxièmement, le diagnostic, et plus particulièrement le diagnostic temporel, constitue une mission importante des bureaux des temps. Il permet la production et l'exploitation de connaissances sur les pratiques temporelles du territoire ${ }^{11}$ dans le but d'orienter les actions et de les adapter aux réalités et aux besoins locaux. Idéalement, le diagnostic est préalable à la mise en œuvre des projets, mais l'analyse de nos études de cas français montre que cela n'est pas toujours possible vu le manque de ressources matérielles et humaines, ou la nécessité d'implanter rapidement des projets et d’obtenir des résultats.

Troisièmement, l'expérimentation renvoie à la mise en œuvre d'initiatives et de projets innovants dans les thématiques citées précédemment. En tant que porteurs et surtout initiateurs de projets, les bureaux des temps s'appuient sur l'expérimentation afin de gagner en crédibilité auprès des décideurs. En effet, lorsque l'implantation de projets-pilotes permet l'accumulation d'expériences et que l'intérêt et la viabilité de ces projets sont démontrés par l'expérimentation, d'autres organismes ou services (dans le cas des collectivités) reprennent la responsabilité et la diffusion de ces projets.

Quatrièmement, le dialogue et la participation sont des composantes essentielles de la mise en œuvre des politiques temporelles. Lune des vocations premières des bureaux des temps est de favoriser la concertation et l'émergence d'un dialogue entre plusieurs acteurs dans des situations de conflits d'usage de l'espace ou du temps. De plus, les limites imposées par les compétences et les moyens des collectivités (par exemple, dans le cas de l'ouverture des commerces ou de la modification des horaires des cours de l'université) les obligent également à établir un dialogue sociétal. Le modèle des tables au minimum quadrangulaires italiennes, regroupant autour d'un sujet de débat commun collectivités locales, société civile, entreprises et salariés, s’applique désormais à presque tous les bureaux des temps et politiques temporelles.
PAR QUELS TYPES D'INTERVENTIONS les villes peuvent-elles favoriser la conciliation travail-famille et le bien-être des citoyens?

Au-delà des actions formelles ou informelles de sensibilisation au bien-être des citoyens, les collectivités, et surtout celles qui se dotées récemment de missions des temps, tendent à adopter les pratiques suivantes.

\section{L'ajustement des temps et de l'organisation du travail, la réduction des disparités sociales et spatiales}

Ces enjeux font référence aux questions d'inégalités de gestion des temps quotidiens entre les hommes et les femmes, dans les ménages et les entreprises. Centrale en Italie et en Espagne, cette thématique est abordée moins directement en Allemagne et aux Pays-Bas. En France, elle s'impose surtout à partir de 2003 (encadré 2).

Cette thématique revêt dans la vie des citoyens une importance accrue par la crise économique depuis 2008. Or, les villes et à plus forte raison les bureaux des temps peuvent difficilement intervenir dans l'organisation des temps et des modes de travail en dehors de la collectivité. Le soutien politique fort apporté à ce type d'initiative, comme lémergence d'une demande émanant des entreprises elles-mêmes (ayant pris la mesure de l'intérêt d'une telle démarche ${ }^{12}$ ), conduit à la multiplication des initiatives favorisant une gestion plus harmonieuse des temps de travail. La Ville de Rennes est ainsi parvenue à modifier l'organisation des temps des agents de propreté au-delà de la collectivité. Une réflexion a cours actuellement dans le Grand Lyon (encadré 3).

\section{Encadré 2}

\section{Les banques de temps}

Les banques des temps constituent une forme d'aide réciproque ou de troc de services permettant à la fois de simplifier la gestion des temps du quotidien et de valoriser les nombreuses activités qui font partie de l'univers familial mais qui ne sont pas monnayables.

Un exemple de ce mécanisme est le cas de la petite commune italienne de Santarcangelo di Romagna, près de Bologne. En 1995, la mairesse et des citoyens ont créé une banque du temps en s'inspirant du modèle existant à Parme. Les membres s'inscrivent sur le fichier central de leur banque du temps en décrivant leurs compétences, au-delà des aptitudes professionnelles. Ils offrent ces compétences aux membres, qui peuvent recourir à leurs services. En même temps, ils définissent leurs besoins, qui pourront être satisfaits par les autres membres. Au moment de l'inscription, ils reçoivent une sorte de chéquier composé de deux parties détachables: I'une est donnée à la personne à laquelle ils destinent leur temps (une demi-heure, une heure, trois heures, etc.) et l'autre est envoyée à la banque du temps. Cette dernière tient le compte des crédits et des débits d'heures pour chaque membre. De telles initiatives ont par la suite été menées en Espagne et en France. 
Une réflexion est menée par le Grand Lyon, la commune de Charly et l'Agence Nationale pour I'Amélioration des Conditions de Travail sur la création d'un centre de télétravail dans le cadre de la réhabilitation d'un domaine, notamment dans le but de réduire le nombre et la durée des déplacements. Selon Lucie Verchère-Tortel, Grand Lyon, mission "temps et services innovants", Direction de la prospective :

«Nous avons été interpellés par le maire d'une commune qui souhaitait réhabiliter une maison bourgeoise. II trouvait dommage de voir les gens de sa commune du sud-ouest de Lyon traverser toute l'agglomération pour aller travailler. II proposait de créer dans sa commune un centre de télétravail où les gens pourraient venir un ou deux jours par semaine. Nous cherchons donc les entreprises qui pourraient être intéressées.

Après, il y a le quartier de la gare Part-Dieu, un quartier en plein devenir et nous aimerions qu'il y ait un lieu de co-travail ou de travail nomade, où I'on souhaite passer si l'on a envie de lancer un projet ou une activité, comme une incubatrice, mais avec une dimension événementielle. Nous en sommes aux études en amont pour voir si des entreprises seraient intéressées, sachant qu'une fois celles-ci découvertes, ce ne serait pas trop difficile."

En dépit de ces avancées, la question de l'égalité hommesfemmes pourtant centrale en Italie demeure la grande oubliée des politiques temporelles en France. On laisse souvent de côté ce chantier difficile, où les progrès se font souvent au coup par coup, au profit d'actions où les résultats sont plus évidents.

\section{L'adaptation de l'offre de services publics et privés aux besoins des citoyens}

L'adaptation des services publics et privés aux besoins et aux rythmes des citoyens implique de revoir les différentes dimensions de l'offre de services publics et privés.

Les horaires d'ouverture et de fermeture des services ainsi que la localisation de ceux-ci continuent de scander nos pratiques $^{13}$. La question des périodes et des horaires d'ouverture des équipements et des infrastructures est d'actualité pour un bon nombre de collectivités. Les piscines, les bibliothèques et médiathèques, les musées, les équipements sportifs, les parcs et les jardins sont concernés au premier chef. Le bureau des temps de Paris et la mission des temps de Montpellier ont été actifs sur ce plan. Ce processus d'harmonisation s'est poursuivi de 2007 à 2010 et d'autres collectivités, telles que les villes de Dijon et de Poitiers, ont mené un travail dans ce sens.

De plus, la répartition des services, privés et publics, est trop souvent inégale sur le territoire. En France comme ailleurs, il faudrait revoir la façon dont ceux-ci sont offerts, par exemple pour favoriser la mise en place de points de regroupement de services. En France, sans avoir donné lieu à une politique à part entière (comme en Allemagne), l'amélioration de la qualité du service public constitue un axe des politiques temporelles (comme en Espagne et aux PaysBas). Cette amélioration se concrétise par la mise en place de dispositifs comme les Points d'information médiation multi services, les maisons de services publics et les guichets uniques (encadré 4).
L'adaptation des services aux besoins des citoyens renvoie également à la capacité des collectivités de jouer un rôle d'incitation auprès des acteurs privés ayant un impact sur loffre et l'organisation des services sur le territoire. À défaut de pouvoir intervenir directement dans ce domaine, les politiques temporelles ont du moins permis aux collectivités d’ouvrir le dialogue avec les acteurs privés (Dijon, Grand Lyon, Paris). Il reste cependant difficile d'influencer l'offre de services privés. Ainsi, si plusieurs marchés alimentaires d'après-midi ont été ouverts à Paris, la discussion amorcée dans ce domaine en 2010 n'a pu aboutir à Dijon.

Le volet concernant l'offre mais surtout l'accessibilité des services publics a été pris en compte dans le cadre des bureaux des temps en France. Ces derniers ont contribué à la mise en place d'espaces multiservices et d'espaces d'aide et d'accueil des usagers contribuant à renforcer à la fois le maillage et la qualité des services sur le territoire. Ces structures, qui ont émergé de 2000 à 2007, se sont multipliées de 2007 à 2010. L’expérience des guichets uniques de rentrée scolaire (Poitiers, Belfort) a été reproduite à Strasbourg en 2010. Des Points d'information médiation multi services ont également été créés à l'initiative des bureaux des temps à Lyon et à Paris, où ils se sont diffusés dans de nombreux arrondissements entre 2007 et 2010. Enfin, une réflexion est en cours à Dijon sur la création de la maison de services publics (déjà existante à Paris).

\section{L'adaptation de l'offre de services d'éducation et de garde aux besoins des citoyens}

Ladaptation des rythmes scolaires. Lécole demeure l'un des plus puissants régulateurs des rythmes individuels et collectifs : "Elle structure la vie des familles qui ont des enfants et celle des enfants eux-mêmes. Elle rassemble plusieurs fois par jour en un même moment et en un même lieu (le parvis de l'école) parents et enfants. Elle rythme les grandes migrations au cours de l'année ${ }^{15}$.» 
Encadré 4

Trois dispositifs pour faciliter l'accès aux services et réduire le temps consacré aux démarches administratives

Les Points d'information médiation multi services sont une structure de proximité mise en place à la suite d'un partenariat entre, d'une part, des services comme EDF (gaz-électricité), France Télécom et La Poste et, d'autre part, I'État et les collectivités locales afin de faciliter l'accès à ces services et, le cas échéant, le règlement de certains litiges, au moyen de la médiation. Ils s'adressent surtout aux populations fragilisées des quartiers urbains. Leur objectif est de favoriser l'autonomie des personnes dans I'utilisation des services publics par une information adaptée et un accompagnement éducatif. Ces points d'information visent un renforcement de l'équité dans l'accès aux services publics et privés.

Les maisons de services publics sont des lieux d'accueil, d'information et d'orientation destinés aux usagers de services publics. Ces structures privées ou publiques sont réparties sur le territoire français (elles existent aussi ailleurs en Europe) et contribuent à une distribution équilibrée des services. Fondées sur un partenariat entre acteurs publics et privés, ces maisons permettent d'élargir la gamme des services offerts en un même lieu. Nées d'expérimentations diverses menées depuis le début des années 1990 en zone rurale comme dans les quartiers urbains dits "sensibles", elles s'inscrivent dans le mouvement de réforme de l'État, qui cherche à rompre avec un modèle bureaucratique d'administration et à rétablir la confiance entre les citoyens et les services publics.

Les guichets uniques de rentrée scolaire s'appuient sur une idée simple : permettre aux parents de régler en peu de temps les démarches liées à la rentrée. Ils constituent un exemple efficace de partenariat entre services publics pour faciliter la vie des usagers.

Par exemple, à Poitiers, le centre d'animation Cap Sud, les services de la ville, la Société des transports poitevins, la caisse d'allocations familiales ainsi que les associations et comités de quartiers saisissent l'idée lancée par l'agence des temps en avril 2001 : réunir en un seul endroit - la Maison de quartier -, à des horaires inhabituels - de $17 \mathrm{~h} 30$ à $19 \mathrm{~h} 30-$, tous les partenaires liés à la rentrée, les associations et les comités de quartiers. En août, une expérimentation est menée dans le quartier de Poitiers Sud.

Les deux guichets uniques ont permis à une centaine de parents l'achat des cartes de cantines, de bus, l'inscription à la bibliothèque, au centre de loisirs, aux activités sportives, I'obtention d'informations, etc. On constate un taux de satisfaction élevé, de la part également de nouveaux habitants qui appréhendent ainsi la richesse de leur quartier.

En 2002, l'expérience a été généralisée à tous les quartiers. Depuis 2003, le public ciblé s'est élargi : au départ, les parents en constituaient le cœur. Aujourd'hui, les étudiants et les adultes s'y intéressent. Les plages horaires ont évolué pour donner plus de possibilités aux pères de s'investir : un guichet unique un samedi matin. Le service Administration générale de Poitiers, dont le "métier" est le service à la population, a pris le relais de l'agence des temps pour l'organisation logistique. En 2007, le service a été étendu sur un septième quartier de Poitiers et un service d'interprétariat a été mis en place pour les personnes sourdes et malentendantes.

Les résultats de cette initiative sont les suivants : le dispositif est ancré dans les habitudes; on observe une augmentation des personnes bénéficiaires (150 par guichet); les familles monoparentales et les femmes cadres supérieures en déficit de temps se sont approprié le dispositif; les pères de familles sont de plus en plus présents, ce qui va dans le sens de l'égalité hommes-femmes; une dynamique s'est créée entre les participants au dispositif ${ }^{14}$.

Les rythmes scolaires et l'idée même de leur modification (leur adaptation aux besoins de l'enfant ou leur révision de manière à faciliter l'organisation du temps des familles) sont une question extrêmement sensible en France comme ailleurs, qui débouche difficilement sur des mesures concrètes même si des réflexions ont déjà été menées sur ce thème au Québec ${ }^{16}$. Les Pays-Bas sont cependant très avancés dans le domaine de la coordination entre l'éducation, l'accueil des enfants et le loisir, grâce à la transformation de certaines écoles en lieux multifonctionnels (les «écoles fenêtres» ou «élargies») ouverts sur leurs milieux de vie (encadré 5).

\section{L'offre de services d'accueil et de garde des enfants}

La qualité et surtout la densité des services d'accueil des
d'Europe à l'autre. Dans ce domaine, la France, comme le Québec, est plutôt avantagée. Cependant, en France comme au Québec, les besoins d'accueil concernant les enfants d'âge préscolaire et la garde selon des horaires atypiques sont encore loin dêtre satisfaits. En France, d'intéressants projets de garde pour les enfants en bas âge ont vu le jour (encadré 6), mais peu de dispositifs concernant les périodes estivale ou hors scolaire ont été créés. L'offre importante d'animation, de garde de jour et de camps de vacances à des coûts abordables proposée par les collectivités et par certains comités d'entreprise explique en partie cet état de fait.

Dans le domaine de laccueil de la petite enfance, les expériences menées à l'initiative des bureaux des temps ou en partenariat avec eux ont connu un réel succès. Elles ont continué à se développer de 2007 à 2010 et ont dans certains cas acquis une autonomie de fonctionnement. Toutefois, la 


\section{Les « écoles élargies " aux Pays-Bas}

La première "école fenêtre » est apparue dans un quartier de Groningue, aux Pays-Bas, en 1996. Aujourd'hui, 88\% des villes du pays possèdent ou sont sur le point de posséder au moins une "école élargie » (il en existe environ 1200 ).

Ce concept d'école fenêtre repose sur trois principes : il s'agit d'établissements qui peuvent accueillir des enfants âgés de 0 à 15 ans; les parents doivent être partie prenante du fonctionnement des établissements; ceux-ci doivent favoriser la possibilité pour les parents de concilier le travail rémunéré et l'éducation des enfants.

Aux Pays-Bas, toutes les écoles, publiques ou privées, sont intégralement subventionnées par l'État. Les écoles élargies ne bénéficient d'aucun financement supplémentaire; il existe cependant des politiques de soutien à l'échelon national. Elles relèvent du niveau local et du niveau non national. Les partenaires locaux déterminent l'organisation de leur école communautaire et les services qui y seront offerts.

Une école fenêtre est constituée d'au moins une école primaire et d'une structure d'accueil de la petite enfance; elle comporte également de nombreuses autres institutions : crèche, bibliothèque, association sportive, école de musique, service social et de santé, etc. Si leur vocation est de prendre en charge les enfants, ces établissements sont aussi ouverts aux parents et aux habitants du quartier.

Le fait de rassembler différents services et activités dans un même immeuble permet de mutualiser le financement de la construction, du fonctionnement et de la maintenance des équipements. L'idéal serait que tous ces services soient réunis dans un même bâtiment, mais environ la moitié des écoles fenêtres fonctionnent à travers la mise en réseau de ces différentes organisations et infrastructures ${ }^{17}$.

\section{Encadré 6}

\section{Le projet "Crèche en gare " de 2005 à 2010}

Les collectivités locales ont compris l'intérêt de développer des zones autour des gares, longtemps délaissées. Ces infrastructures deviennent I'enjeu d'une politique urbaine qui combine le transport multimodal, l'emploi, les bureaux, I'habitat et les commerces. En septembre 2005, neuf partenaires ${ }^{18}$ ont signé un plan d'action visant à favoriser l'intermodalité pour l'aire urbaine lyonnaise. Le projet de Réseau Express de l'Aire métropolitaine Lyonnaise (REAL) gravite autours du Transport Express Régional (TER) et prévoit développer l'offre de transport, aménager les correspondances entre les réseaux et offrir des services en gare.

La mise en place des crèches à proximité des gares périurbaines ou dans celles-ci fait partie de la deuxième phase de la démarche Réseau Express de I'Aire métropolitaine Lyonnaise (REAL 2) engagée en 2010 par les partenaires du réseau. L'objectif est d'aménager les abords des gares pour ensuite offrir une bonne qualité de services sur les grands axes de transports collectifs. Ces actions ont également pour but de rendre les transports collectifs compétitifs par rapport à l'automobile, notamment pour les déplacements quotidiens. Le périmètre d'intervention englobe 80 gares, dont 33 situées dans l'agglomération lyonnaise. Une microcrèche à la gare de Roanne a d'ailleurs ouvert ses portes en 2010. Cet établissement d'une capacité de 9 berceaux propose un accueil régulier et occasionnel aux enfants âgés de 10 semaines à 4 ans. Si les usagers du TER sont privilégiés, le service reste ouvert à tous les publics. Fruit d'un partenariat étroit entre diverses collectivités, cette crèche représente un projet-pilote dans le vaste plan de redynamisation des gares lancé par la SNCF (Société nationale des chemins de fer français). Quatorze autres gares devraient être concernées dans la région. Par exemple, on pourrait imaginer la mise sur pied de telles garderies dans les gares du réseau métropolitain de Montréal pour faciliter la vie des parents banlieusards qui travaillent au centre-ville.

réflexion sur l'évolution ou l'adaptation des rythmes scolaires évolue très peu, étant contrainte par la tendance à la réduction de l'effectif du personnel éducatif et enseignant.

\section{L'adaptation de l'offre de services de transport et de mobilité aux besoins des citoyens}

La mobilité, et plus particulièrement la mobilité durable, constitue également un enjeu reconnu très tôt par les instigateurs des politiques temporelles. Elle joue un rôle déterminant dans les problématiques de l'accessibilité des services, des infrastructures, de l'emploi, du développement durable (en relation avec les émissions de gaz à effet de serre et la qualité de la vie), de l'égalité des personnes et des territoires, en particulier dans un contexte d'augmentation des distances et de la durée des déplacements quotidiens ${ }^{19}$. La question est importante au Québec également, et notamment à Montréal, où les banlieues ne cessent de sétendre, ce qui allonge les temps de déplacement des parents-travailleurs.

Dans ce contexte, les politiques temporelles, grâce à leur rôle de médiation et à leur capacité de mettre en relation les acteurs clés de ce domaine, favorisent la mise en place de 
systèmes de déplacement partagés. Les plans de déplacement en entreprises (Montpellier) et interentreprises (Grand Lyon) constituent un exemple de solution ainsi qu'une réelle avancée (encadré 7). Ils sont également répandus sous des formes voisines dans d'autres pays européens (comme les pactes de mobilité en Allemagne et en Italie).

De 2007 à 2010, l'engagement des bureaux des temps dans le domaine de la mobilité a été croissant. La question de la mobilité renvoie à des pratiques de déplacement individuelles et collectives entre lesquelles il est nécessaire de réaliser des arbitrages pour favoriser une meilleure articulation. Or, les actions menées dans ce domaine impliquent un processus de concertation entre de nombreux acteurs. La mise en ouvre de ces plans et la concertation qu'elle nécessite se matérialisent le plus souvent par létablissement de plans de déplacements à différentes échelles : en entreprise, interentreprises ou urbains.

\section{L'adaptation de l'offre de services aux besoins des travailleurs de nuit ou de soir}

La nuit est longtemps restée un espace-temps en friche et appréhendée comme une discontinuité, le temps du sommeil et, par extension, l'arrêt de toute activité ${ }^{20}$. Dans le cadre des politiques temporelles, il est de plus en plus fréquent de traiter de la question de la nuit plutôt que d'un territoire de 24 heures. Ce refus de l'élargissement à l'infini des horaires constitue également l'orientation prise par l'Allemagne et l'Italie. Pourtant, comment ne pas prendre en compte la nuit urbaine, ses rythmes et ses différents usages, incluant le travail de nuit pour certains salariés?

Lobjectif poursuivi par les politiques temporelles est de faciliter la conciliation entre ceux qui dorment, s'amusent ou travaillent dans leur diversité (jeunes étudiants ou non, salariés, exclus, etc.). Les actions menées par les bureaux des temps dans le domaine de l'animation nocturne semblent les plus fragiles. En effet, l'animation proposée par les collectivités ne correspond pas toujours aux attentes des citoyens et elle est de plus relativement coûteuse.

En revanche, la question de la mobilité nocturne et, plus encore, celle de la conciliation et de la qualité de la vie nocturne, intégrant une dimension animation, semblent en plein développement. Si Belfort n’a pu maintenir son service de bus de nuit destiné aux jeunes, Paris, Lyon, Dijon et Poitiers ont, avec la participation de leurs bureaux des temps, renforcé leur offre de transports de nuit. Par ailleurs, le développement de la concertation et des dispositifs visant à améliorer la conciliation des différents usages de la nuit et de la ville s'est affirmé, de 2007 à 2010, par la signature de chartes (Paris, Caen, Dijon, Saint-Denis), la mise en place du système de correspondant de nuit et le déroulement des états généraux de la nuit (Paris, 12 et 13 novembre 2010).

\section{L'adaptation de l'aménagement urbain ou territorial aux besoins des citoyens}

La question de la rénovation et de la réaffectation des espaces urbains fait également partie des enjeux unanimement relevés par les porteurs des politiques temporelles. Les projets concernent généralement la revitalisation des espaces urbains (France et Italie). C'est d'ailleurs dans cette optique qu'a été mis en place le projet de crèche en gare que nous avons décrit. Il s'agit de favoriser la multifonctionnalité des lieux, domaine dans lequel s'illustrent les Pays-Bas ${ }^{21}$ et l'Allemagne. Comme l'exprime avec humour Royoux ${ }^{22}:$ «Envisager le temps comme moyen d'articuler les différentes échelles de laction publique (notamment en matière d'aménagement) constitue presque une petite révolution copernicienne».

Dans le champ de l'urbanisme, l'action des bureaux des temps est encore limitée. Cependant, les actions entreprises et l'insertion de la logique temporelle dans les documents d'urbanisme tendent à faire passer l'action des bureaux des temps de la rénovation urbaine (Saint-Denis, Rennes) à une démarche se rapprochant de l'urbanisme temporel.

Encadré 7

Des outils pour la mise en œuvre d'une mobilité durable : l'exemple des plans de déplacements interentreprises dans le Grand Lyon

Dans le Grand Lyon, 10 plans de déplacements interentreprises ont été mis sur pied depuis 2007, touchant environ 400 entreprises et plus de 20000 salariés. II s'agit d'une démarche collective et participative, dans laquelle les acteurs volontaires d'une zone d'activité économique (salariés, direction de l'entreprise, clients, fournisseurs) se concertent en vue de proposer des mesures qui faciliteront leurs déplacements pour accéder à leur lieu de travail.

Sur chaque territoire, le projet est soutenu par les entreprises, à travers les associations d'entreprises, et le financement est assuré à $80 \%$ par des fonds publics et à $20 \%$ par les entreprises.

Plusieurs raisons incitent les entreprises à s'engager dans la démarche d'un plan de déplacements interentreprises : cela permet de limiter le nombre de voitures et de places de parking, de réduire le budget consacré aux transports pour les salariés, de rendre plus fluide l'accès au lieu d'activité, de rendre le territoire plus attrayant, d'agir pour l'environnement en limitant la pollution, de se regrouper de manière à peser dans les négociations avec les autorités chargées de l'organisation des transports. Le fait de réunir plusieurs entreprises permet d'élaborer des outils communs, de partager des solutions et de répartir les frais, quand on décide, par exemple, de se doter d'une flotte de vélos. 
En France comme en Europe, la multiplication des expériences découlant directement ou indirectement des bureaux des temps montre que, en dépit de leurs difficultés à s'engager sur ces terrains, les collectivités locales sont parfaitement conscientes de l'importance de ces enjeux. On mesure donc à la fois le potentiel des politiques temporelles et l'immense défi qui leur est lancé. Nous dégagerons, à partir de l’analyse de la capacité des bureaux des temps et de leurs initiatives à s'inscrire dans la durée, lévolution des enjeux liés aux temps et les conditions de succès des expériences favorisant l'amélioration de la conciliation des temps sociaux.

À léchelle locale, les bureaux des temps compensent la faiblesse de leur marge de manœuvre par un travail considérable de sensibilisation interne et externe, allant jusquà intégrer les questions temporelles à leurs projets d’aménagement et à leurs documents de planification urbaine. Toutefois, à l'heure actuelle, la mise en place des bureaux des temps est souvent plus proche de la fonction d'incubateur de projets temporels que d'une véritable politique temporelle globale. Pourtant, sans masquer le caractère encore modeste de l'action des bureaux des temps, la multiplication des projets temporels et la récurrence des thématiques traitées révèlent l'importance sociale des problématiques temporelles, comme l'indique la montée de la problématique de la conciliation travail-famille au Québec (Tremblay, 2012) et la mise en place d'une Agence des temps dans la MRC Charlevoix-est.

\section{CONDITIONS DE SUCCÈS}

\section{et limites des interventions des villes}

La réussite des expériences des bureaux des temps se traduit à la fois par leur inscription dans la durée, leur diffusion et leur reproduction d'une collectivité à l'autre, l'augmentation au sein d'une collectivité du nombre de bénéficiaires et l’expression de la satisfaction des bénéficiaires.

Certains éléments constituent des facteurs de succès pour la mise en œuvre des politiques familiales municipales : la nomination des responsables des questions familiales (soit une ressource spécifiquement désignée), l’expression de la volonté politique (un soutien explicite des élus) et létablissement de partenariats avec les acteurs locaux ${ }^{23}$. Force est de constater que ces conditions sont également indispensables au succès des bureaux des temps et de leurs initiatives.

\section{Obtenir des ressources et l'appui des élus}

Le travail de communication, de diagnostic et de montage de projets comportant de nombreux acteurs nécessite un investissement incompressible en temps de la part de la personne chargée de la mise en place du bureau des temps. Ainsi que l'exprime un participant de Montpellier : «En arrivant en poste, j’ai fait la tournée des villes qui avaient établi un bureau ou une agence des temps : je l'ai bien vu, tous les bureaux des temps qui nont pas accordé de moyens humains suffisants (au moins une personne à temps plein ou presque) ont échoué ou disparu.»

De même, les changements culturels et organisationnels que supposent les initiatives touchant au rythme et à l'organisation des activités et des services requièrent dêtre relayés aux élus locaux et soutenus par eux. Selon un participant de Montpellier : «Les bureaux des temps où cela a le mieux fonctionné avaient, sur le plan politique, des personnalités fortes pour les soutenir et les mettre en œuvre.»

Enfin, les bureaux des temps doivent susciter l'engagement de divers acteurs au sein de la collectivité où ils sont implantés, des autres échelons administratifs territoriaux et du milieu local, et ce, pour trois raisons. En premier lieu, vu sa vocation transversale, le bureau des temps n'est pas conçu pour mener seul des projets; il doit en effet attirer la participation de l'ensemble des services de sa collectivité. Selon un participant de Caen : «Le bureau des temps ne travaille sur des dossiers qu'en collaboration avec les services, ce qui permet de démultiplier les moyens; c'est aussi une richesse.» De son côté, un participant de Dijon déclare : «Il n’est pas nécessaire (ni souhaitable?) que ce soient les temps urbains qui réalisent les projets. Il est nettement préférable que les collègues des autres services s'investissent.»

En deuxième lieu, pour pouvoir mettre en place des projets d'envergure ou ayant un caractère multidimensionnel, il importe de faire appel à des compétences ou à des ressources externes. Selon un expert de Saint-Denis : «Tout le monde sait aujourd'hui que l'on ne fait rien sans des financements croisés et que les compétences s'entremêlent largement.»

En troisième lieu, le partenariat peut permettre aux bureaux des temps de toucher le secteur privé et de parvenir à faire participer des entreprises en se gardant de toute ingérence. Le cas du plan de déplacements interentreprises à Lyon est à ce titre révélateur.

\section{CONCLUSION}

On peut faire un bilan relativement positif des politiques temporelles. En dépit de quelques échecs et abandons d'activités, les projets temporels semblent en mesure de s'inscrire dans le moyen terme et de trouver au sein de la société locale les conditions de leur maintien. Cependant, bien que les bureaux des temps soient des facilitateurs et des initiateurs de projets et de modes d'organisation innovants, ils ne semblent pas en mesure de porter à eux seuls cette évolution. Il est nécessaire que d'autres acteurs à la fois publics (l'État, les autres échelons territoriaux, les ministères responsables de l'éducation, de la famille, des municipalités ou des transports) et privés (les entreprises, les technoparcs, etc.) soient sollicités et s'associent à l'action. 


\section{NOTES}

1. Ministère de l'Emploi, de la Solidarité sociale et de la Famille (2004 : 284).

2. $\operatorname{CDRFO}(2003,2004)$.

3. Rochman et Tremblay (2010a, 2010b).

4. Un projet d'agence des temps est actuellement en développement dans la région de Charlevoix, en lien avec le Carrefour Action municipale et famille.

5. Voir Gagnon et Guérin (2011)

6. Boulin (2003).

7. Boulin (2003).

8. S'il existe différentes conceptions de la conciliation travail-famille, il est le plus souvent admis que celle-ci " consiste en un ensemble de mesures organisées et d'actions pour faciliter l'articulation des responsabilités familiales et sociales, professionnelles et de formation et pour permettre le développement harmonieux des membres de la famille, ainsi qu'un cheminement professionnel continu pour les travailleuses et les travailleurs" (Béchard, 2004).

9. Boulin (2008)

10. Pour plus d'information, voir le site http ://tempoterritorial.free. fr/spip.php ?rubrique1.

11. Soit les caractéristiques des horaires des services, des entreprises, des transports, de la durée du travail, des besoins en matière de temps, de l'accessibilité, des déplacements (provenance, destination, motifs), etc.
12. Lee-Gosselin (2005).

13. Godard (2003).

14. Source : http : //qualite-publique.org/Les-Guichets-uniques-aPoitiers.html ?id_rubrique=7, consulté le 5 novembre 2010.

15. Godard (2003).

16. Conseil de la famille et de l'enfance (2006).

17. Source : Boulin (2011); Europa, I'alliance européenne pour les familles, http : //ec.europa.eu/employment_social/emplweb/ families/index.cfm ?langld=fr\&id=5\&pr_i_id=147, consulté le 10 avril 2011.

18. Le Grand Lyon, la Région Rhône-Alpes, Saint-Étienne Métropole, les Conseils généraux du Rhône, de l'Ain et de l'Isère, les Communautés d'agglomération de Villefranche, de Porte d'Isère et du pays Viennois, ainsi que la SNCF, Réseau Ferré de France et le SYTRAL (Syndicat Mixte des Transports pour le Rhône et l'Agglomération Lyonnaise).

19. Boulin, entrevue de septembre 2010.

20. Gwiazdzinski (2005).

21. Voir notamment l'expérience des écoles communautaires (ou élargies) aux Pays-Bas et en Allemagne et développée notamment par Boulin (2008) et par Baaijens (2010).

22. Royoux, entrevue de 2010.

23. Observatoire québécois du loisir (2004).

\section{RÉFÉRENCES}

Baaijens, C. (2010), "Les écoles élargies : 15 années de mutualisation d'équipements scolaires aux Pays-Bas ", Actes des Temporelles 2010, Aménagement urbains temporels, 14-15 octobre.

Béchard, C. (2004), "Concilier travail-famille : où sont nos priorités comme société?", mémoire pour la consultation publique : "Pour une politique gouvernementale sur la conciliation travailfamille ", AFEAS, http ://bv.cdeacf.ca/CF_PDF/84406.pdf, consulté en septembre 2009.

Boulin J.-Y. (2003a), co-directeur d'ouvrage avec F. Godard et P. Dommergues, La nouvelle aire du temps. Editions de l'Aube.

Boulin, J.-Y. (2008), Villes et politiques temporelles, La Documentation Française.

Boulin, J.-Y. (2011), "Des écoles ouvertes sur le quartier", Ade/s Revue Territoires, $\mathrm{n}^{\circ}$ 516, mars, p. 40-43, www.adels.org/ territoires/516.htm, consulté le 10 avril 2011.

CDRFO (Conseil de développement de la recherche sur la famille du Québec) (2003), Dossiers "Médiation familiale " et "Politiques sociales", vol. $4, n^{\circ} 4$.

CDRFO (Conseil de développement de la recherche sur la famille du Québec) (2004), Dossier "Famille et municipalités", vol. 5, n 1.

Conseil de la famille et de l'enfance (2006), Les familles, l'école et son service de garde : un projet éducatif partagé?

Gagnon, M.-È., Guérin, D. (2011), L'Agence de Charlevoix-Est, un bureau des temps typiquement québécois, Développement social, vol. $12, \mathrm{n}^{\circ} 1$, juin, www.revueds.ca/lagence-des-temps-dela-mrc-de-charlevoix-est-un-bureau-des-temps-typiquementquebecois.aspx, consulté le 12 décembre 2012.
Godard, Francis (2003), "Les Français et le temps dans la ville ", l'État de l'opinion, Éditions du Seuil.

Godard, Francis, (2002), "Cessons d'opposer temps individuels et temps collectifs ", Ceras - revue Projet $n^{\circ} 273$, Mars. URL : http : // www.ceras-projet.com/index.php ?id=1702.

Gwiazdzinski, L. (2005), La nuit dernière frontière de la ville, Éditions de l'Aube.

Lee-Gosselin, H. (2005), " Pourquoi des entreprises mettent en place des mesures de conciliation emploi-famille?", dans Tremblay, D.G. (dir.), De la conciliation emploi-famille à une politique des temps sociaux, Presses de l'Université du Québec.

Ministère de l'Emploi, de la Solidarité sociale et de la Famille (2004), Vers une politique gouvernementale sur la conciliation travailfamille, document de consultation.

Observatoire québécois du loisir (2004), "La famille... c'est aussi l'affaire des municipalités", vol. 2, $n^{\circ} 1$.

Rochman, J., Tremblay, D.-G. (2010a), Politiques familiales municipales (PFM) et développement local : les PFM comme outil de soutien aux stratégies locales au Québec, notes de recherche n²010-6 de la l'ARUC sur la gestion des âges et des temps sociaux, ARUCGATS, www.teluq.uqam.ca/aruc-gats.

Rochman, J., Tremblay, D.-G. (2010b), Politiques familiales municipales : l'émergence de nouvelles formes de gouvernance locale?, notes de recherche $n^{\circ}$ 2010-7 de la l'ARUC sur la gestion des âges et des temps sociaux, ARUC-GATS, www.teluq.uqam. ca/aruc-gats. 